\title{
Investigation of Circular Flexural Hinge Used in Compliant Mechanism Using FEA Tool.
}

\author{
${ }^{I}$ M.M.Sawant and ${ }^{2}$ P.R.Anerao \\ ${ }^{1,2}$ Vishwakarma Institute of Information Technology, Kondhawa (Bk),Pune Maharashtra, India \\ Imilindsawant77@gmail.com
}

\begin{abstract}
To reduce fatigue failure of compliant mechanism, it is necessary to design and analyze the flexure hinge parametrically. A methodology to design a flexural hinges for compliant mechanism is proposed in this paper to improve fatigue life. Results obtained by finite element analysis shows that used design equations are reliable and easier to be used in the design of such proportion flexural hinges. The proposed analytical model gives a new viewpoint on the design of circular flexure hinge based compliant mechanisms. Circular flexural joint was manufactured by using Al $6061 \mathrm{~T} 6$ material and experimental setup is developed to test this flexural hinge. Results obtained by FEA were found to be in good correlation with experimental results. The variation in the results can be attributed to variation in properties of material, actual dimensions of setup etc.
\end{abstract}

Index Terms- Bending stress, compliant mechanism, deflection, fatigue failure, flexural hinge.

\section{INTRODUCTION}

Compliant mechanisms offer the deformation with their flexible members to transfer motion, force, and energy. It serves attractive benefits in the sense of manufacturing and performance over traditional rigid link mechanisms. The wide range of applications requires compact flexible structures that exhibit specific functional features different from traditional rigid mechanisms. Usage of compliant mechanisms has been increased extensively in precision positioning applications such as precision X-Y stages [1], piezoelectric motors [2], high accuracy alignment devices for optical fibers, active dampers [3], high-precision cameras, scanning tunneling microscopes, robotic microdisplacement mechanisms. Due to the low displacement of the piezoelectric actuator; flexure hinges are used to amplify the displacement amplitude. Compliant mechanism are being popular due to various advantages like one piece manufacturing (monolithic constructions), zero backlashes, reduced weight, no need of lubrication, smoothness of movement and virtually infinite resolution. When flexure hinges are used in a mechanism, the static and dynamic characteristics of mechanism depends mostly on the performance of the flexure hinge [4]. The Performance evaluation of the flexural hinge is done by systematically investigating the effect of geometric parameters on the performance characteristics like deformation, stiffness; stress; motion range and accuracy [5] of the flexure hinges. Paros and Weisbord [6], in their primary work presented the design equations for calculating the compliances of both single-axis and two-axis circular cross-section flexural hinges. Ragulskis et al. [7] applied the static finite element analysis to single-quarter of circular flexural hinges for calculating their compliances. Results of this analysis were further used to formulate a criterion based on the deformation of an incipiently straight cross-section; which allows specifying optimal flexural geometry for minimum bending stress and stiffness. Lobontiu et al. [8] developed an analytical model of circular flexure hinges that are included into planar amplification mechanisms. Compliance factors were formulated for evaluating the precision of motion, rotation efficiency, precision of motion and stresses. $\mathrm{Xu}$ and King [9] performed static finite element analysis of circular, elliptic and cornerfilleted flexure hinges. The results shows that the elliptical flexural hinge has less stress for the similar displacement, the corner filleted flexural hinge was the most accurate in the sense of motion; while the right circular flexural hinge has more stiffness. Ryu and Gweon [10] modeled the motion errors that are induced by machining imperfections into a flexural hinge mechanism. Hwang [11] proposed an in-plane $\mathrm{X}-\mathrm{Y}-\theta$ micropositioning stage with piezoelectric actuators based on Taguchi design method. A flexure based compliant displacement amplification mechanism is studied according to the 
proposed analytical method. Tian et al [12] described the mechanical design and dynamics of a 3DOF flexure-based parallel mechanism. Dirksen et al [13] worked on fatigue life of flexural hinges, by analytical expressions considering stress concentration, non-zero mean stresses, surface finish factors and multiple loading conditions. Dirksen and Lammering [14] used energy principles to calculate the desired properties at individual points within hinges and proposed valuable closed-form equations of circular, parabolic shaped flexure hinges. In this study it is found that circular geometry of flexural hinges yields moderately high rotational deflection and bending stiffness, while the center of rotation remains close to the center point of the hinge. Shuib et al. [15] concluded that, for some compliant structures design requirements may be many millions of life cycles but usually it fails at flexural hinges before completing his life cycles. Premature or unexpected failure in the device can result in unsafe design. Hence, it is necessary to critically analyze and determine the fatigue life of a compliant mechanism. It is also noted that there were too little of previous results on the analysis of fatigue failure in compliant mechanisms.

The integration of different functions into fewer parts offers great advantages, but it also requires the simultaneous design for motion and forces of flexural hinge. Another challenge is fatigue life which needs to be addressed for most compliant mechanisms because, their motion comes from repeatedly bending of flexible parts due to which stresses are induced in to the flexural parts in turns it undergoes fatigue failure. In this paper analysis of fatigue failure for circular flexural hinge is carried out at static and dynamic conditions. Methodology for calculating fatigue life and deflection of circular flexural hinge made of Al 6061 T6 was developed and simulated with standard package of FEA, also experimental set up is prepared for validating the design.

\section{ANALYTICAL CALCULATION}

The influence of the hinge geometry on the deformation behavior of flexural hinges has been investigated for several forms, which affect the performance of path generation of the mechanism [7]. The deflection, equivalent stress and fatigue life are calculated by providing a constant ratio of hinge height to neck height, the notch sensitivity, surface finish factor, size factor. Flexure hinges are sensitive to temperature variation due to thermal expansion and contraction, so a factor of thermal expansion is also taken into the consideration.

\subsection{Characteristics and Other Parameters of Flexural hinge}

Analytical expressions are derived by using the standard X-Y coordinate system as shown in Figure1. Location of the notch is at the center of a hinge having a length of L. One end of hinge is fixed while the load is applied at another end, like in cantilever beam. Ratio $t / R$ is considered as 0.25 for satisfactory fatigue life conditions. This ratio describes stiffness or strength of flexural hinge. Failures occur in the notched area of flexural hinges due to reduced cross section. Thus, notch sensitivity factor is important in the design of flexural hinges. Flexure hinge characterized by length L, depth $b$, height $\mathrm{H}$, neck thickness $\mathrm{t}$ and common points P1, P2, P3 to resist external load Fy, or moment My as illustrated in figure 1.

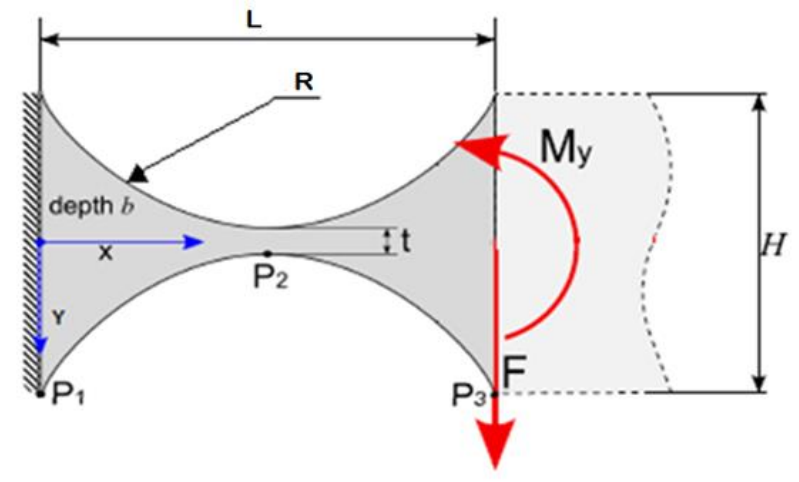

Figure 1 Planar flexure hinge 


\subsection{Equations}

The applied force causes angular deflection in hinge; Paros and Weisboard derived the compliance ratio equation with the help of beam theory $[7,8]$.

$\frac{\alpha_{z}}{M_{Z}}=\frac{9 \pi(R)^{\frac{1}{2}}}{2 E b(t)^{\frac{5}{2}}}$

Above equation is simplified in term of stress as,

$\sigma_{\max }=\frac{8}{\pi} \times \frac{\mathrm{Fl}^{2}}{\mathrm{~b}(\mathrm{R})^{\frac{1}{2}}(\mathrm{t})^{\frac{5}{2}}}$

Equation (2) is rearranged into obtain force after substituting compliance ratio $t / R=0.25$

$\mathrm{F}=\frac{\pi}{4} \times \frac{\sigma_{\max } \times \mathrm{b}}{\mathrm{l}^{2}} \times \mathrm{t}^{3}$

Material properties of aluminum alloy $\mathrm{Al} 6061$ t6 used in compliant mechanisms are listed in the table below. $[10,11,12,13]$.

Table 1 Mechanical Properties of aluminum alloy Al 6061 T6

\begin{tabular}{cc}
\hline Mechanical Properties & Al 6061 T6 \\
\hline Ultimate Stress $\left(\mathrm{S}_{\mathrm{ut}}\right)$ & $310 \mathrm{Mpa}$ \\
Yield Stress $\left(\mathrm{S}_{\mathrm{yt}}\right)$ & $276 \mathrm{Mpa}$ \\
Young's Modulus $(\mathrm{E})$ & $73 \mathrm{Gpa}$ \\
Min. tensile Strength $\left(\mathrm{S}_{\mathrm{t}}\right)$ & $300 \mathrm{Mpa}$ \\
\hline
\end{tabular}

The fatigue strengths of flexural hinges are calculated using stress-life approach. For next calculations of notched hinge factors like stress concentration, notch sensitivity, surface finish etc. have been considered which affects the fatigue life and deflection.

\subsubsection{Endurance limit stress $S_{e}$}

A. Approximate endurance limit

$\mathrm{S}_{\mathrm{e}}=0.5\left(\mathrm{~S}_{\mathrm{ut}}\right)$

B. Surface finish factor

$\mathrm{K}_{\mathrm{a}}=\mathrm{a} \times\left(\mathrm{S}_{\mathrm{t}}\right)^{\mathrm{b}}$

\section{Size factor $K_{b}$}

It is a considered that $95 \%$ area is going under the stress, this area is known as effective area.

Effective area $d_{e}$ 
$d_{e}=\sqrt{\frac{A_{95}}{0.0766}}$

$\mathrm{A}_{95}=(0.05 \times \mathrm{b} \times \mathrm{t})$

For bending and torsion the equation is in following form

For $2.79 \mathrm{~mm}<\mathrm{d}<51 \mathrm{~mm}$

$\mathrm{K}_{\mathrm{b}}=1.24 \times \mathrm{d}_{\mathrm{e}}^{0.107}$

D. Stress concentration factor $K_{t}$ for semicircular notch.

The fatigue strength of notched flexural hinge is significantly lower than for the unnotched flexural specimen. So stresses are concentrated at the notch of the flexural hinge, causing nucleation of micro cracks which possibly leads to failure. The stress concentration factor $\mathrm{K}_{\mathrm{t}}$ is used to account for the occurring higher stresses,

$\mathrm{K}_{\mathrm{t}}=3.065-6.637\left(\frac{2 R}{H}\right)+8.229\left(\frac{2 R}{H}\right)^{2}-3.636\left(\frac{2 R}{H}\right)^{3}$

Notch sensitivity factor

$q=\frac{1}{1+\sqrt{\frac{a}{R}}}$

$\sqrt{a}=$ Neuber's constant

$\mathrm{K}_{\mathrm{f}}=1+\mathrm{q} \times(\mathrm{Kt}-1)$

\section{E. Modified stress concentration factor}

$\mathrm{K}_{\mathrm{d}}=\frac{1}{\mathrm{~K}_{f}}$

Endurance limit stress $S_{\mathrm{e}}$

It accomplishes by using equations (3),(4),(5),(6).

$\mathrm{S}_{\mathrm{e}}=\left(\mathrm{K}_{\mathrm{a}} \times \mathrm{K}_{\mathrm{b}} \times \mathrm{K}_{\mathrm{c}} \times \mathrm{K}_{\mathrm{d}} \times \mathrm{S}^{\prime}{ }_{\mathrm{e}}\right)$

\subsubsection{Stresses in flexural hinges}

Flexural hinges are mainly used in compliant mechanism to allow rotational motion, thus the focus is on axial bending caused by external force $\mathrm{F}$ in the $\mathrm{y}$ direction, and the $\mathrm{M}_{\mathrm{y}}$ moment, as illustrated in Fig.1. All loads are assumed to be time-harmonic with constant amplitude and mean.

Moment of area

To calculate the bending stresses in flexural hinges, moment of area is required and is calculated by following process.

Area $=\mathrm{b} \times \mathrm{t}$ 
And moment of inertia

$\mathrm{I}=\mathrm{b} \times \mathrm{t}^{3} / 12$

Therefore, Induced stress is,

$\sigma_{\max }=\frac{M}{I} \times y$

\subsubsection{Fatigue life using basquin's equation}

Basquin proposed the mathematical equation to represent the part of the $\mathrm{S}-\mathrm{N}$ curve for the region $\mathrm{N}$ $>10^{6}$

$\mathrm{a}=\mathrm{S}_{f} \times N^{b}$

$\mathrm{S}_{\mathrm{f}}=\frac{F \times L \times 6}{b \times t^{2}}$

$\mathrm{a}$ and $\mathrm{b}$ are constant.

\subsubsection{Deflection accomplish in the flexural hinge.}

Deflection in the direction of force is,

$\Delta y=\frac{9 \pi}{2 E b} \times\left(\frac{R}{t}\right)^{\frac{5}{2}} \times F$

This equation (7) was developed using Euler-Bernoulli beam equation and Timoshenko's beam theory $[6,15]$. By using such analytical expiration the parameters are obtained by providing constant length and force parameters.

Table 2:- Dimensions obtained by analytical approach

\begin{tabular}{ll}
\hline Parameters & $\begin{array}{l}\text { Dimensions } \\
(\mathrm{mm})\end{array}$ \\
\hline Length & 60 \\
Neck thickness & 3.21 \\
Radius of curvature & 12.84 \\
Height of hinge & 28.96
\end{tabular}

\section{FEM ANALYSIS}

The FEM analysis was used as a strong tool for stress analysis in elastic parts of compliant mechanisms. Aim of the FEA is to verify that the stresses induced are under the elastic limit of the chosen material. The input parameters for FEA are: material properties of Al 6061 t6 which are mentioned in table 1. The dimensions of the model are illustrated in table 2. Load and boundary condition applied are shown in figure 2. The hinge is fixed from one end and a load of $10 \mathrm{~N}$ is applied to another end. This FEM analysis has been conducted in standard FEA package Ansys 14.5. 


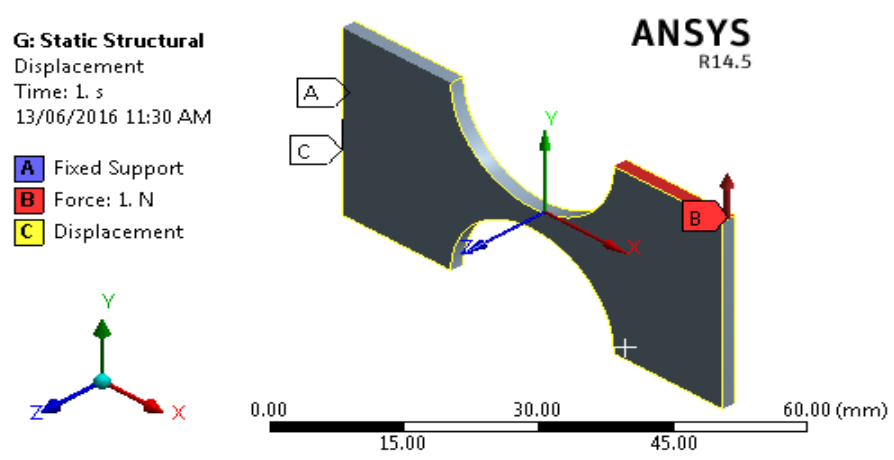

Figure 2 Model of Circular flexural hinges.

Fatigue failure of the flexible elements of compliant mechanisms can occur due to tension, compression, torsion or bending. But bending, and sometimes torsion are the dominant considerations. Here, the main focus is on fatigue failure of flexible elements of compliant mechanisms due to bending. Fatigue failure due torsion or combined stresses can be treated in a similar manner. In bending, fatigue life will be closely correlated with the maximum stress which is a function of the deflection and moment of inertia of the beam. To lower induced stress and increase fatigue life, moment of inertia is to be reduced appropriately. This is done by making the beam thinner. It should be noted that as the moment of inertia is reduced the stiffness of the beam is also reduced, changing the response of the beam to loadings. This bending effect is illustrated in the figure 3 and 4 in term of stress, deflection, and fatigue life.

G: Static Structural

Equivalent Stress

Unit: MPa

10/06/2016 7:43 PM

$\square \begin{aligned} & \text { 62.212 Max } \\ & 55.299\end{aligned}$

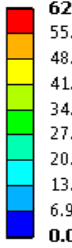

55.299

48.387

41.475
34.562

27.65

20.738

13.825
6.9129

0.00055403 Min
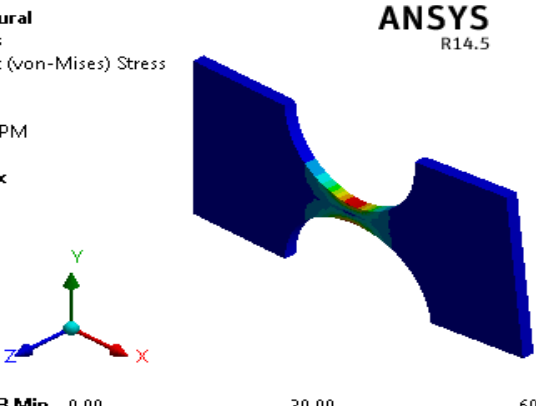

Figure 3 Distribution of stress
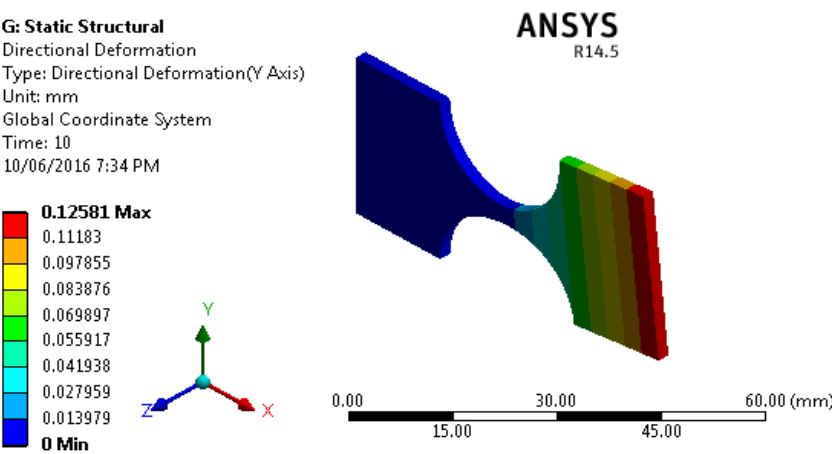

Figure 4 Directional deformation of flexure
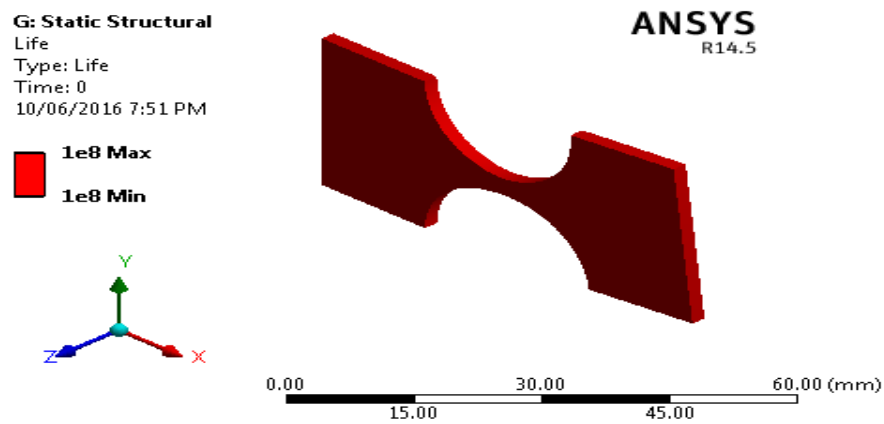

Figure 5 Infinite life cycles achieved at design load conditions in flexural joints

\section{Experimental Investigation and Result Discussion}

The flexible flexural hinge is manufactured by using water jet cutting machine (WJCM) based on derived geometrical parameters. Manufactured hinge is shown in figure 6. The details of experimental setup used in study are shown in figure 7. Linear motorized actuator with the motor driver having the 
displacement range 0 to $5 \mathrm{~mm}$, load cell of $3 \mathrm{Kg}$ working with 9 to $12 \mathrm{v}$ voltage. LVDT of 0 to 100 $\mathrm{mm}$ with signal amplifier used to measure hinge deflection. Cyclic load is applied using stepper motor to the flexural member and load cell is placed in between motor and flexural member to measure applied load. LVDT is placed at the free end of the hinge to record output deflection. Similarly, the input deflection, strain, and output displacement were recorded in amplification unit with respective to force change.

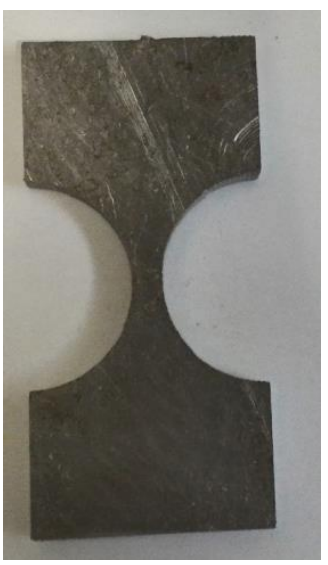

Figure 6 specimen

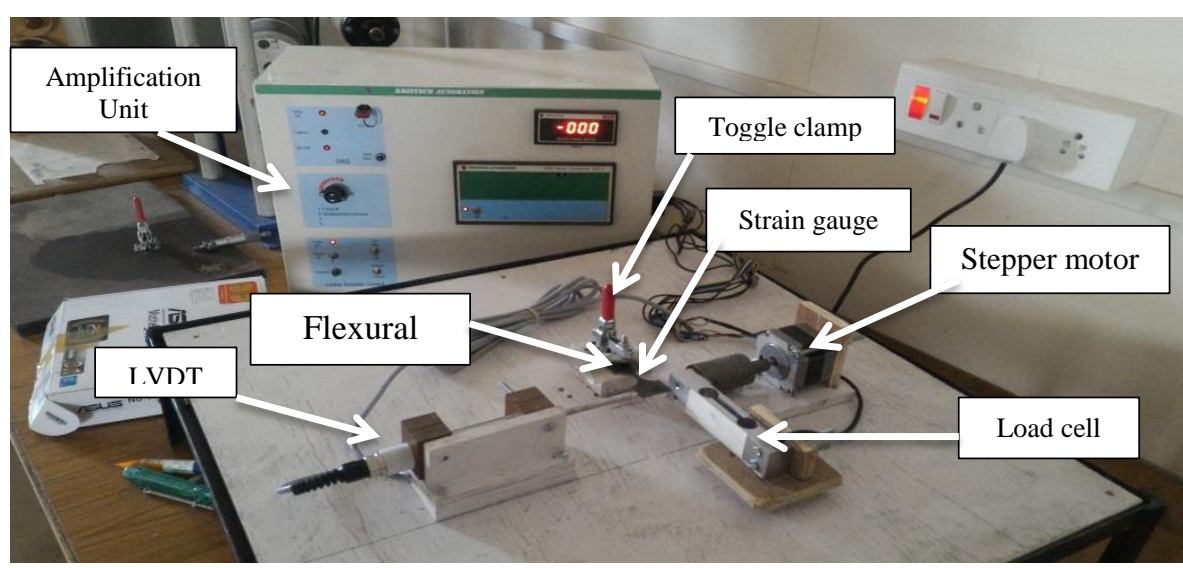

Figure 7 experimental setup

Tests have been carried at different forces with constant length and neck thickness. Effect of cyclic loading conditions on stress and displacement events are described graphically in figure 8 and 9. Induced stress is less than yield limit of the material under consideration which is the indication of safe design with a factor of safety 1.5. Thus, the component is having an infinite fatigue life.

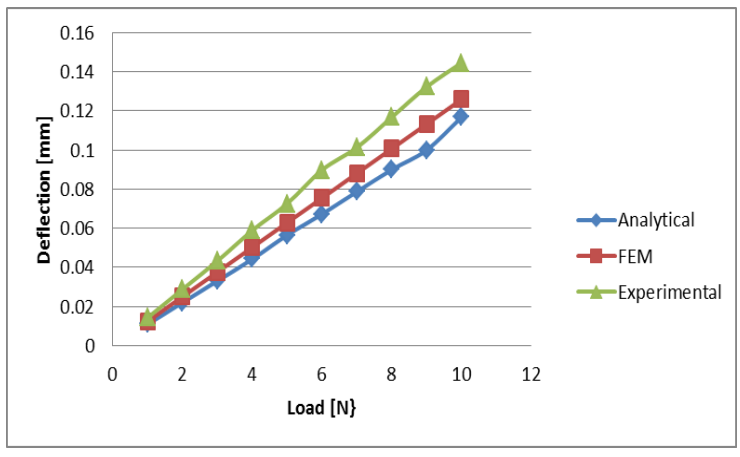

Figure 8 Displacement vs. Load Results

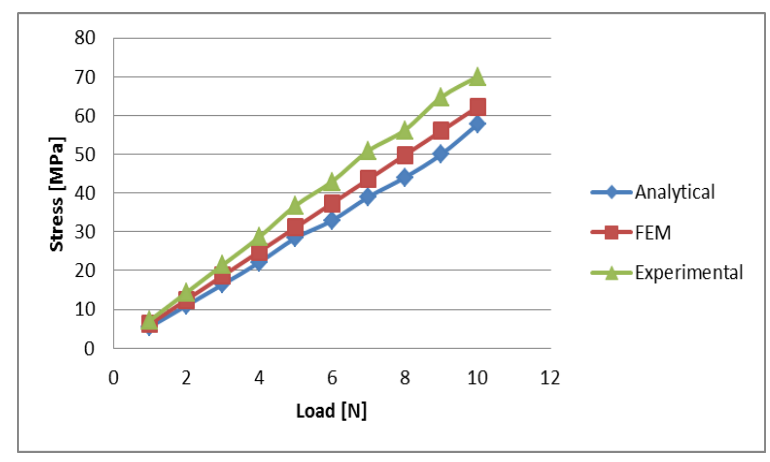

Figure 9 Stress vs. Load Results

\section{CONCLUSION}

To avoid the fatigue failure of flexural hinges in the compliant mechanism, critical analysis of flexural joint is required. Some factors like compliance ratio, stress concentration, notch sensitivity etc. affecting the failure are discussed in this paper. The methodology is presented for designing a circular flexural hinge to improve the fatigue life. The analysis of designed circular flexural hinge is carried on Al6061 T6 material. Results by Analytical, FEM, and experimental approaches are gained and compared on the basis of deflection and induced stresses. It is observed that the easiest way to achieve deflection in simple compliant mechanism configurations is by reducing the moment of inertia of the 
flexural element in bending. This approach has great impact on the fatigue failure of flexural elements. The other way to achieve deflection is by increasing the length of the element in bending. This will increase the deflection for a given loading without increasing stress levels but this will result in lower fatigue life of flexure elements.

\section{Nomenclature:}

$\mathrm{t} / \mathrm{R}$ Compliance ratio

$t \quad$ Neck thickness of the hinge

$\mathrm{R}$ Radius of curvature

$\mathrm{b}$ Width of the hinge

L length of hinge

$\mathrm{H}$ height of hinge

F applied load

E Young's modulus

$\mathrm{K}_{\mathrm{a}} \quad$ Surface finish factor

$\mathrm{K}_{\mathrm{b}} \quad$ Size factor

$\mathrm{K}_{\mathrm{t}}$ Stress concentration factor

$\mathrm{K}_{\mathrm{d}}$ Modified stress concentration factor

$\Delta y$ Deflection in y directions

$\sigma_{\max }$ Max Stress induced

$\mathrm{S}_{f} \quad$ Fatigue strength

$\mathrm{N}$ No. of cycles

\section{REFERENCES}

[1] Moriyama, S., Harada, T. and Takanashi, A., "Precision X-Y stage with a piezo-driven fine-table", Bull Japan Soc Precision Engineering,22, 13-14,1988.

[2] Ishida, M., Hamaguchi, J., Shirasuka, K. and Hori, T., "A new friction-type piezoelectric motor utilizing mechanism of the strain wave gearing", IEEE Trans Ind Electronics,.39, 30-35,1992.

[3] Galvagni J., "Electrostrictive actuators and their use in optical applications", Opt Engineering, 29, $1389-1391,1990$.

[4] Dongwoo Kang and Daegab Gweon, "Analysis and design of a cartwheel-type flexure hinge", Precision Engineering, 37, 33-43, 2013.

[5] Brian P. Trease, Yong-Mo Moon and Kota S, "Design of large-displacement compliant joints", Journal of Mechanical Design, 127, 788-798, 2004.

[6] Paros J.M., Weisbord L., "How to design flexure hinges", Machine Design, 25, 151-156,1965.

[7] Ragulskis K.M., Arutunian M.G., Kochikian, A.V., Pogosian, M.Z., "A study of fillet type flexure hinges and their optimal design", Vibration Engineering, 3, 447-452.1989.

[8] Lobontiu N., Paine J.S.N., Garcia E., Goldfarb M., "Corner filleted flexure hinges", ASME Journal of Mechanical Design, 123, 346-352, 2001.

[9] Xu, W., King, T.G., "Flexure hinges for piezo-actuator displacement amplifiers: flexibility, accuracy and stress considerations", Precision Engineering, 19, 4-10, 1996.

[10] Ryu J.W., Gweon D.G., "Error analysis of a flexure hinge mechanism induced by machining imperfection", Precision Engineering, 21, 83-89 1997.

[11] Hwang, J. Byun, J. Jeong and M. G. Lee "Robust design and performance verification of an inplane xy micropositioning stage". IEEE Transactions on Nanotechnology, 10(6), 1412-1423, 2011.

[12] Tian, B. Shirinzadeh and. Zhang D "Design and dynamics of a 3-dof flexure-based parallel mechanism for micro/nano manipulation". Microelectronic Engineering, 87(2) 230-241, 2010.

[13] Dirksen F, Anselmann Mathias, Zohdi T. I., Lammering R "Incorporation of flexural hinge fatigue-life cycle criteria into the topological design of compliant small-scale devices" Precision Engineering, 37 (3), 531-541, 2013. 
INTERNATIONAL JOURNAL OF ENGINEERING TECHNOLOGY AND SCIENCES (IJETS) Vol.5 (1) June 2016 DOI: http://dx.doi.org/10.15282/ijets.5.2016.1.5.1044

[14]. Dirksen F and. Lammering R, "On mechanical properties of planar flexure hinges of compliant mechanisms", Mechanical Science, 2, 109-117 2011.

[15] Kramer S. N. Saxena A. "A simple and accurate method for determining large deflections in compliant mechanisms subjected to end forces and moments Transactions" ASME Journal of Mechanical Design, 120, 392-400, 1998.

[16] Solehuddin Shuib, Ridzwan M.I.Z and Kadarman A H., "Methodology of Compliant Mechanisms and its Current Developments in Applications: A Review", American Journal of Applied Sciences vol 4(3), 159-166, 2007. 